\title{
THE EFFECT OF X-IRRADIATION ON CHIASMA FREQUENCY IN CHORTHIPPUS BRUNNEUS
}

\author{
M. WESTERMAN* \\ Department of Genetics, University of Birmingham, England
}

Received 20.viii.70

\section{INTRODUGTION}

THE formation of chiasmata during or immediately prior to pachytene in eukaryotic cells can no longer be thought of as an isolated event. The weight of evidence to date suggests that it is the end-product of an interrelated series of processes initiated at some earlier stage of meiosis, some or all of which can be affected by a variety of physical and chemical agents. In the desert locust, Schistocerca gregaria, Westerman (1967) has shown that following exposure of males to a single dose of X-rays there are three discrete periods at which chiasma frequency may be altered. Thus irradiation during the Sphase of spermatogonial mitoses and during leptotene-early zygotene gave a significant increase in chiasma frequency when scored at diplotenediakinesis. In contrast, an equal dose given during premeiotic DNA synthesis led to a significant decrease.

These alterations in mean cell chiasma frequency in $S$. gregaria appear to be the result of alterations in the number of chiasmata formed by the long and medium bivalents only, the three small bivalents continuing to form a single chiasma under all conditions. Since the chromosomes of this species, though all telocentric, do not respond in the same way, it seemed worth while extending the study to another grasshopper species, Chorthippus brunneus (Thunb.) - one which includes metacentric as well as telocentric elements in its complement.

\section{MAterial AND MEthods}

In the first of two duplicate experiments, 87 fifth instar and young adult male Chorthippus brunneus from a laboratory culture of animals originating from the wild were divided into three groups. All individuals from two of these groups were injected abdominally at time zero with about $1.25 \mu \mathrm{Ci}$ of ${ }^{3} \mathrm{H}$-thymidine in $0.03 \mathrm{ml}$. distilled water (Thymidine-6T $(n) 5.00 \mathrm{Ci} / \mathrm{mM}$ R.G.C. Amersham). One of these two labelled groups was then immediately given a dose of $150 \mathrm{r}$. X-rays (for details see Westerman, loc. cit.). The experiment thus consisted of a control group, a labelled control group and an irradiated labelled group of insects. All three groups were subsequently maintained in an incubator at $30^{\circ} \mathrm{C}$. and fed fresh grass daily. At appropriate time intervals (table 1, A) insects were taken at random from each group, their testes removed by vivisection and fixed in $1: 3$ acetic alcohol.

In the second experiment, 31 young adult males were injected with the same amount of ${ }^{3} \mathrm{H}$-thymidine and irradiated with $150 \mathrm{r}$. X-rays as described above. These animals were then maintained in the same incubator at

\footnotetext{
* Present address: Department of Genetics, La Trobe University, Melbourne, Australia.
} 
$30^{\circ} \mathrm{C}$. and individuals sacrificed at random at appropriate time intervals (table I, B).

TABLE 1

The number of individuals sampled at each fixation time

Fixation time in days post-injection

$\begin{array}{ll} & \text { Experiment } \\ \text { Control } & \\ \text { A Control }+\mathrm{H}^{3} \text {-Thymidine } \\ 150 \mathrm{r}+\mathrm{H}^{3}-\text { Thymidine } \\ \text { B } 150 \mathrm{r}+\mathrm{H}^{3} \text {-Thymidine }\end{array}$

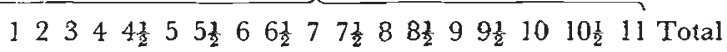

$\begin{array}{llllllllllllllllllll}2 & 2 & 2 & 2 & 2 & 2 & 2 & 2 & 1 & 1 & 2 & 2 & 1 & 2 & 1 & 1 & 11 & 1 & 28\end{array}$

$\begin{array}{llllllllllllllllllll}2 & 2 & 1 & 2 & 2 & 2 & 2 & 2 & 1 & 1 & 2 & 2 & 1 & 1 & 1 & 1 & 0 & 0 & 25\end{array}$

$\begin{array}{lllllllllllllllllll}2 & 2 & 2 & 2 & 2 & 2 & 2 & 2 & 1 & 2 & 2 & 2 & 1 & 2 & 2 & 2 & 2 & 2 & 34\end{array}$

$\begin{array}{llllllllllllllllllll}0 & 2 & 1 & 0 & 0 & 2 & 2 & 2 & 2 & 2 & 2 & 3 & 3 & 2 & 2 & 2 & 2 & 2 & 31\end{array}$

Prior to scoring, each individual was coded and randomised and chiasma frequencies were scored from lacto-propionic orcein stained squash preparations made from a part of each testis. Where possible, chiasma scores were recorded at diplotene-diakinesis for 20 cells per individual. In experiment A, three of the irradiated individuals, one from each of days $9 \frac{1}{2}, 10$ and $10 \frac{1}{2}$ post-irradiation, contained no suitable cells.

TABLE 2

Mean cell chiasma score $\pm \overline{\mathrm{sd}}$ for each sampling time shown in table 1

$\begin{array}{lcccccc}\begin{array}{c}\text { Time } \\ \text { in } \\ \text { days }\end{array} & \text { Or } & \text { Or }+\mathrm{H}^{3} & \text { Control } & 150 \mathrm{r} . \mathrm{A}+\mathrm{H}^{3} & \mathrm{I} 50 \mathrm{r} . \mathrm{B}+\mathrm{H}^{3} & 150 \mathrm{r} . \\ +1 & 13 \cdot 60 \pm 0 \cdot 1783 & 14 \cdot 35 \pm 0 \cdot 1913 & 13 \cdot 98 \pm 0 \cdot 1366 & 14 \cdot 35 \pm 0 \cdot 1810 & - & 14 \cdot 35 \pm 0 \cdot 1810 \\ +2 & 13 \cdot 95 \pm 0 \cdot 1992 & 13 \cdot 40 \pm 0 \cdot 1747 & 13 \cdot 68 \pm 0 \cdot 1352 & 14 \cdot 38 \pm 0 \cdot 1114 & 14 \cdot 97 \pm 0 \cdot 2337 & 14 \cdot 65 \pm 0 \cdot 1280 \\ +3 & 13 \cdot 78 \pm 0 \cdot 2163 & 12 \cdot 50 \pm 0 \cdot 1701 & 13 \cdot 35 \pm 0 \cdot 1728 & 14 \cdot 66 \pm 0 \cdot 1777 & 14 \cdot 75 \pm 0 \cdot 1619 & 14 \cdot 67 \pm 0 \cdot 1783 \\ +4 & 13 \cdot 40 \pm 0 \cdot 1518 & 13 \cdot 23 \pm 0 \cdot 1409 & 13 \cdot 31 \pm 0 \cdot 0951 & 13 \cdot 70 \pm 0 \cdot 1527 & - & 13 \cdot 70 \pm 0 \cdot 1527 \\ +4 \frac{1}{2} & 13 \cdot 75 \pm 0 \cdot 1670 & 13 \cdot 68 \pm 0 \cdot 2160 & 13 \cdot 71 \pm 0 \cdot 1357 & 13 \cdot 45 \pm 0 \cdot 1289 & - & 13 \cdot 45 \pm 0 \cdot 1289 \\ +5 & 13 \cdot 50 \pm 0 \cdot 1861 & 13 \cdot 43 \pm 0 \cdot 1957 & 13 \cdot 46 \pm 0 \cdot 1342 & 12 \cdot 88 \pm 0 \cdot 2184 & 14 \cdot 00 \pm 0 \cdot 1661 & 13 \cdot 36 \pm 0 \cdot 1577 \\ +5 \frac{1}{2} & 12 \cdot 73 \pm 0 \cdot 1640 & 13 \cdot 03 \pm 0 \cdot 1735 & 12 \cdot 88 \pm 0 \cdot 1198 & 13 \cdot 98 \pm 0 \cdot 1660 & 13 \cdot 38 \pm 0 \cdot 1589 & 13 \cdot 68 \pm 0 \cdot 1190 \\ +6 & 14 \cdot 60 \pm 0 \cdot 2449 & 13 \cdot 25 \pm 0 \cdot 1670 & 13 \cdot 70 \pm 0 \cdot 1600 & 13 \cdot 45 \pm 1 \cdot 1430 & 13 \cdot 85 \pm 0 \cdot 1542 & 13 \cdot 65 \pm 0 \cdot 1069 \\ +6 \frac{1}{2} & 13 \cdot 35 \pm 0 \cdot 2326 & 12 \cdot 45 \pm 0 \cdot 3033 & 12 \cdot 90 \pm 0 \cdot 2019 & 13 \cdot 60 \pm 0 \cdot 2103 & 13 \cdot 98 \pm 0 \cdot 1621 & 13 \cdot 85 \pm 0 \cdot 1298 \\ +7 & 14 \cdot 30 \pm 0 \cdot 2626 & 13 \cdot 00 \pm 0 \cdot 2294 & 13 \cdot 65 \pm 0 \cdot 2011 & 13 \cdot 45 \pm 0 \cdot 1474 & 13 \cdot 70 \pm 0 \cdot 1485 & 13 \cdot 58 \pm 0 \cdot 1049 \\ +7 \frac{1}{2} & 13 \cdot 05 \pm 0 \cdot 1859 & 12 \cdot 88 \pm 0 \cdot 1901 & 12 \cdot 96 \pm 0 \cdot 1325 & 12 \cdot 93 \pm 0 \cdot 1804 & 13 \cdot 15 \pm 0 \cdot 1318 & 13 \cdot 04 \pm 0 \cdot 1117 \\ +8 & 13 \cdot 85 \pm 0 \cdot 1542 & 13 \cdot 83 \pm 0 \cdot 1822 & 13 \cdot 84 \pm 0 \cdot 1210 & 12 \cdot 38 \pm 0 \cdot 1463 & 13 \cdot 00 \pm 0 \cdot 1008 & 12 \cdot 75 \pm 0 \cdot 0892 \\ +8 \frac{1}{2} & 13 \cdot 75 \pm 0 \cdot 2392 & 12 \cdot 70 \pm 0 \cdot 2626 & 12 \cdot 23 \pm 0 \cdot 1944 & 12 \cdot 20 \pm 0 \cdot 2471 & 12 \cdot 25 \pm 0 \cdot 1053 & 12 \cdot 24 \pm 0 \cdot 0994 \\ +9 & 13 \cdot 40 \pm 0 \cdot 1281 & 12 \cdot 75 \pm 0 \cdot 2161 & 13 \cdot 18 \pm 0 \cdot 1176 & 13 \cdot 28 \pm 0 \cdot 1386 & 13 \cdot 63 \pm 0 \cdot 1325 & 13 \cdot 45 \pm 0 \cdot 0973 \\ +9 \frac{1}{2} & 13 \cdot 30 \pm 0 \cdot 2524 & 13 \cdot 55 \pm 0 \cdot 1697 & 13 \cdot 43 \pm 0 \cdot 1514 & 12 \cdot 65 \pm 0 \cdot 1817 & 13 \cdot 35 \pm 0 \cdot 1412 & 13 \cdot 12 \pm 0 \cdot 1191 \\ +10 & 12 \cdot 45 \pm 0 \cdot 2348 & 15 \cdot 13 \pm 0 \cdot 2950 & 13 \cdot 21 \pm 0 \cdot 2971 & 13 \cdot 35 \pm 0 \cdot 1301 & 13 \cdot 90 \pm 0 \cdot 1710 & 13 \cdot 72 \pm 0 \cdot 1566 \\ +10 \frac{1}{2} & 13 \cdot 70 \pm 0 \cdot 2065 & - & 13 \cdot 70 \pm 0 \cdot 2065 & 12 \cdot 60 \pm 0 \cdot 2225 & 13 \cdot 35 \pm 0 \cdot 1500 & 13 \cdot 10 \pm 0 \cdot 1317 \\ +11 & 14 \cdot 00 \pm 0 \cdot 2177 & - & 14 \cdot 00 \pm 0 \cdot 2177 & 13 \cdot 25 \pm 0 \cdot 1708 & 13 \cdot 30 \pm 0 \cdot 1485 & 13 \cdot 28 \pm 0 \cdot 1125\end{array}$

A further part of the testis from each labelled individual was used to obtain autoradiographs from Feulgen stained squash preparations (Kodak AR 10 stripping film). After a 30-day exposure period, the autoradiographs were developed in Kodak D19b and fixed in Amfix.

\section{Results}

\section{(a) Meiotic timing}

The first wave of labelled cells to arrive at diplotene-diakinesis in experiment A was seen in one of the two irradiated individuals sampled on day $7 \frac{1}{2}$. 
All injected animals sampled on days 8 and $8 \frac{1}{2}$ post-injection from both irradiated and control groups showed label over all meiotic stages from pachytene to anaphase II with the grains being distributed over both homologues. With the single exception of the individual noted above, no other injected animal sampled on or prior to day $7 \frac{1}{2}$ had label over any postzygote stage. In the samples taken on day 9, the only labelled cells were either pre-pachytene or spermatid in nature. Thus, in both irradiated and control groups of experiment A meiosis takes 8 to $8 \frac{1}{2}$ days. Of this time prepachytene stages occupy 6-6 $\frac{1}{2}$ days, premeiotic DNA synthesis up to 1 day and the two meiotic divisions 1 day. Since this is true of both $150 \mathrm{r}$.treated and unirradiated individuals, it would appear that the X-ray treatment had no significant delaying effect on meiosis. These meiotic timings were confirmed by analysis of the labelling patterns in experiment $B$.

TABLE 3

Analyses of variance of the chiasma frequency data

$\begin{array}{lllll}\text { Item } & \text { d.f. } & \text { S.S. } & \text { M.S. } & \text { V.R. }\end{array}$

1. Between treatments

(a) Orv Or $+H^{3}$ Thymidine

2. Between times

3. Interaction

$\begin{array}{lll}1 & 14.1097 & 14 \cdot 1097\end{array}$

$15 \quad 119.6362 \quad 7.9757$

$15 \quad 135.6004 \quad 9.0400$

$<1>0.20$

4. Between individuals within times within treatments

5. Between cells within individuals

9

$\begin{array}{rrr}19 & 343.0101 & 18.0532 \\ 957 & 852.1649 & 0.8905\end{array}$

(b) $150+A$ v $150+B$

1. Between treatments

2. Between times

3. Interaction

4. Between individuals with times and treatments

5. Between cells within individuals

$\begin{array}{rrr}1 & 33 \cdot 1941 & 33 \cdot 1941 \\ 14 & 417 \cdot 9661 & 29 \cdot 8547 \\ 14 & 39.7154 & 2 \cdot 8368 \\ & & \\ 26 & 174.5313 & 6 \cdot 7127 \\ 1032 & 859.6508 & 0.8330\end{array}$

$\begin{array}{cl}4.9449 & 0.05 * \\ 4.4475 & <0.001^{* * *} \\ <1 & >0.20 \\ & \\ 8.0585 & <0.001 * * *\end{array}$

$1032 \quad 859 \cdot 6508 \quad 0 \cdot 8330$

\section{(b) Chiasma scores}

The standard karyotype of Chorthippus brunneus has been fully described elsewhere (John and Hewitt, 1966; Southern, 1967). In addition to a single allocyclic X chromosome, the male complement consists of 16 autosomes. On the basis of length and centromere position these can be placed in two distinct groups, one consisting of three pairs of metacentric chromosomes $\left(\mathrm{L}_{1}-\mathrm{L}_{3}\right)$, the other consisting of five pairs of telocentric chromosomes $\left(\mathrm{M}_{4}-\mathrm{M}_{7}\right.$ and $\mathrm{S}_{8}$ ). Because of the practical difficulty of consistently distinguishing between the $L_{1}$ and $L_{2}$ bivalents and between the $M_{4}$ and $M_{5}$ bivalents, chiasma frequency values were recorded separately for the six chromosome groups $\mathrm{L}_{1+2}, \mathrm{~L}_{3}, \mathrm{M}_{4+5}, \mathrm{M}_{6}, \mathrm{M}_{7}, \mathrm{~S}_{8}$. The results are summarised in table 2 .

Since it was not always possible to score the requisite 20 cells from each individual, the analyses of the data have been carried out in terms of a weighted least squares analysis using KDF9 computer. In each analysis only those times common to both treatments can, of course, be used, since only these contribute to the interaction items. 
Two controls, Or and $\mathrm{Or}+\mathrm{H}^{3}$-Thymidine were included in the experimental design to facilitate the detection of any effect on chiasma frequency ascribable to the $\mathrm{H}^{3}$-Thymidine injection alone. A comparison of these two controls is shown in table $3(a)$. When tested against the appropriate estimate of error (item 4), none of the three main items of this analysis proves to be significant. Tritiated thymidine alone, therefore, has no detectable effect on mean cell chiasma frequency and so the two controls were pooled. The significance of item 4 when tested against item 5 in the above analysis is to be expected, since this is a measure of the heterogeneity of the genotypes of the individuals used in the experiment.

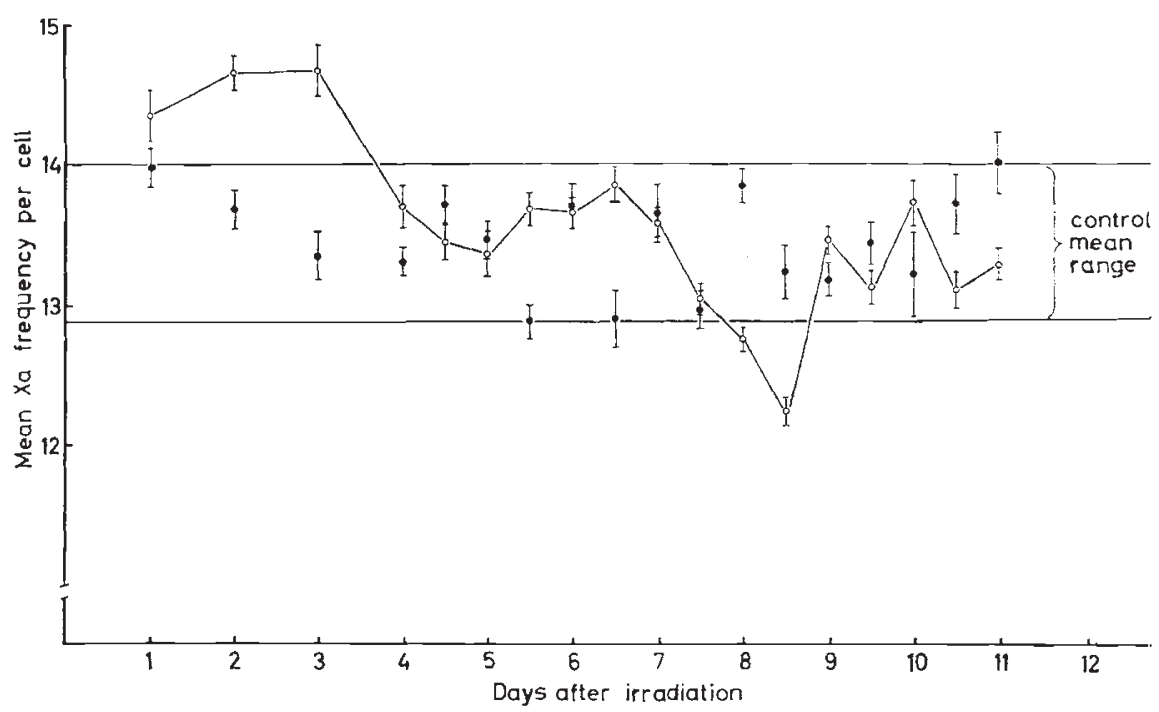

FIG. 1.-Change in chiasma frequency with time. Means and standard deviations are given for irradiated $\bigcirc$ and for control data - Overall control mean $\pm 13.45 \pm 0.374$.

Table $3(b)$ shows a comparison of the two irradiated groups of individuals. Although both the between treatments (item 1) and the between times (item 2) main effects appear to be significant, the former is only just so (VR $=4.9449$ $\mathrm{P}=0.05$ ). It is important to note that the interaction item (item 3 table $3(b))$ is not significant. Thus although the chiasma scores in experiment B tend consistently to be slightly higher than in experiment A (see table 2), the absence of any significant interaction item indicates that both groups are behaving in the same way with time. The difference between the mean value for each treatment is probably simply a reflection of the different histories of the batches of insects used in each experiment. Since the patterns of response with time were similar for these two groups, as were the meiotic timings, the two $150 \mathrm{r}$. treatments were pooled.

The net effect of $150 \mathrm{r}$. X-rays compared with the control is shown in fig. 1 and table 4. Only the between times item (item 2) is significant when tested against item $4(\mathrm{VR}=1.8825 \mathrm{P}=0.05-0.01)$. The results of the analysis shown in this table suggest that not only do the $150 \mathrm{r}$. treatment and the control not differ from one another in mean cell chiasma frequency but that they both react in the same way with time. This result can probably be 
attributed to the large size of item 4 M.S. in the table, since when the control and irradiated groups were analysed separately above, a different conclusion was reached. That the X-ray treatment does indeed affect mean cell chiasma frequency is supported by the results of a series of $t$-tests shown

TABLE 4

Analysis of variance of the pooled control and pooled irradiated data

\section{Item}

1. Between treatments

2. Between times

3. Interaction

4. Between individuals within times within treatments

5. Between cells within individuals

\section{d.f.}

4.8717

$315 \cdot 5779$

$17 \quad 235 \cdot 7575$

79

$779 \cdot 0262$

$2140 \quad 1817 \cdot 5807$
M.S.

V.R.

$\begin{array}{rc}4 \cdot 8717 & <1 \\ 18.5634 & 1 \cdot 8825\end{array}$

$13.8681 \quad 1.4063$

$9 \cdot 8611$

$11 \cdot 6109$

$0 \cdot 8493$

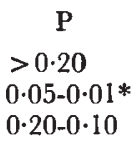

$<0.001^{* * *}$

in table 5. Here the mean of each time of the $150 \mathrm{r}$. treatment shown in fig. 1 is compared with the overall control mean.

Two main points emerge from fig. 1 and table 5. Firstly there is a significant increase $(\mathrm{P}<0.001)$ in mean cell chiasma frequency over the control mean on days 1,2 and 3 post-irradiation, and secondly there is a significant reduction of mean cell chiasma frequency, also at the 0.001 level

TABLE 5

The direction of significant deviations in mean cell chiasma frequency between the irradiated samples and the pooled control sample

$\begin{array}{crc}\begin{array}{c}\text { Day Post- } \\ \text { treatment }\end{array} & t & n \\ 1 & 4 \cdot 7 & 1086 \\ 2 & 8 \cdot 4 & 1121 \\ 3 & 6 \cdot 6 & 1090 \\ 4 & 1 \cdot 3 & 1086 \\ 4 \frac{1}{2} & <1 \cdot 0 & 1086 \\ 5 & <1 \cdot 0 & 1116 \\ 5 \frac{1}{2} & 1 \cdot 7 & 1126 \\ 6 & 1 \cdot 5 & 1126 \\ 6 \frac{1}{2} & 2 \cdot 5 & 1106 \\ 7 & <1 \cdot 0 & 1126 \\ 7 \frac{1}{2} & 3 \cdot 0 & 1126 \\ 8 & 5 \cdot 7 & 1146 \\ 8 \frac{1}{2} & 8 \cdot 8 & 1126 \\ 9 & <1 \cdot 0 & 1126 \\ 9 \frac{1}{2} & 2 \cdot 0 & 1106 \\ 10 & 1 \cdot 7 & 1106 \\ 10 \frac{1}{2} & 2 \cdot 2 & 1106 \\ 11 & 1 \cdot 2 & 1126\end{array}$

$\begin{array}{cc}\mathrm{P} & \begin{array}{c}\text { Direction of } \\ \text { deviation }\end{array} \\ \ll 0 \cdot 001 & + \text { ve } \\ \ll 0 \cdot 001 & + \text { ve } \\ \ll 0 \cdot 001 & + \text { ve } \\ 0 \cdot 20-0 \cdot 10 & - \\ >0 \cdot 30 & - \\ >0 \cdot 30 & - \\ 0 \cdot 10-0 \cdot 05 & - \\ 0 \cdot 20-0 \cdot 10 & - \\ 0 \cdot 02-0 \cdot 01 & + \text { ve } \\ >0 \cdot 30 & - \\ 0 \cdot 01-0 \cdot 001 & - \text { ve } \\ \ll 0 \cdot 001 & - \text { ve } \\ \ll 0 \cdot 001 & - \text { ve } \\ >0 \cdot 30 & - \\ 0 \cdot 05-0 \cdot 02 & - \\ 0 \cdot 10-0 \cdot 05 & - \\ 0 \cdot 05-0 \cdot 02 & - \\ 0 \cdot 30-0 \cdot 20 & -\end{array}$

on days 8 and $8 \frac{1}{2}$ post-treatment. Chiasma scores were made on cells at diplotene-diakinesis and since the most advanced labelled cells on days 1 , 2 and 3 were at leptotene-early zygotene, then by extrapolation from the meiotic timings above, these cells showing an increased chiasma frequency were at late zygotene-early pachytene at the time of irradiation. This finding, though not seen in the $S$. gregaria experiment, is none the less in 
agreement with the results of Lawrence $(1961 a, b)$ following $\gamma$-irradiation of Lilium longiflorum and Tradescantia paludosa.

The decrease on days $8-8 \frac{1}{2}$ coincides with the arrival of labelled cells at diplotene-diakinesis. Thus as in previous experiments, irradiation of cells undergoing premeiotic DNA synthesis leads to a reduction in chiasma frequency. It is interesting to note that the single individual sampled on day $8 \frac{1}{2}$ in experiment $B$ which exhibited a more advanced labelling pattern than others taken at the same time also had a higher chiasma frequency

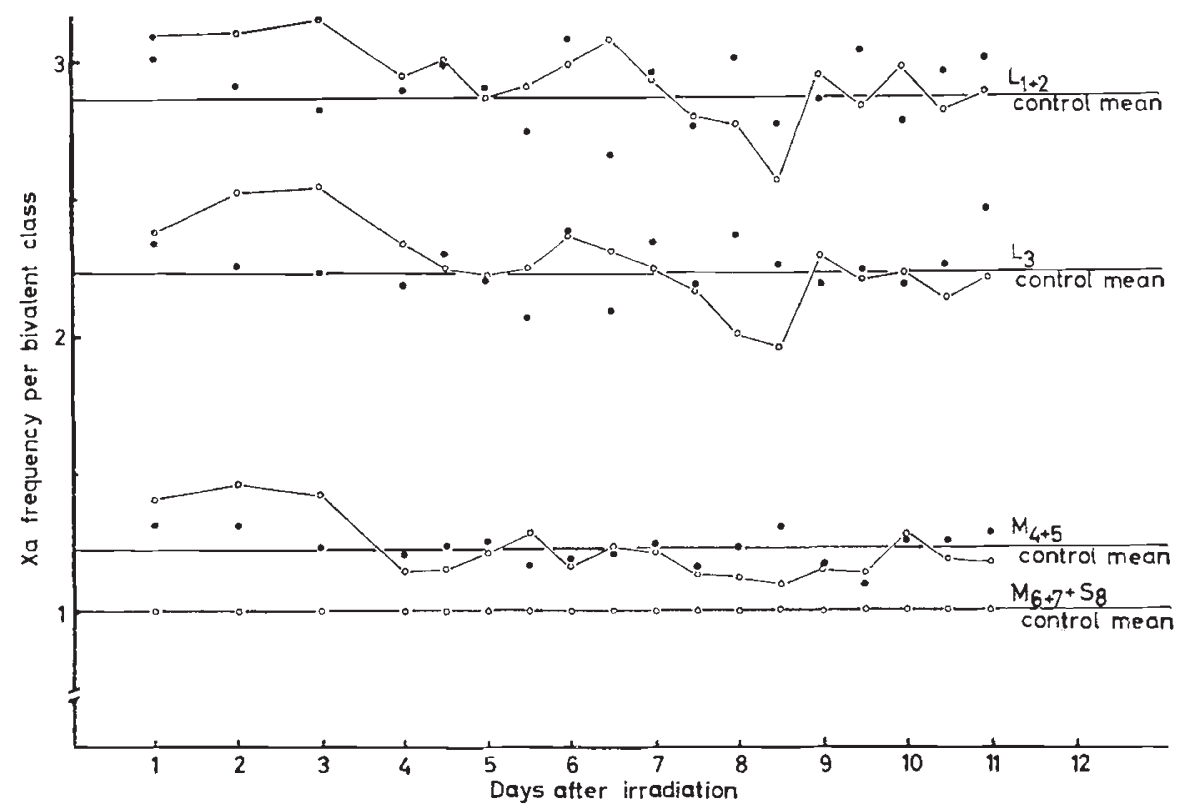

FIG. 2.- The response of the four different classes of bivalent to irradiation (Irradiated data O——, control data $-\bullet)$.

(12.70; cf. 12.20 and $11 \cdot 85)$. Similarly the individual showing labelled diplotene cells on day $7 \frac{1}{2}$ in experiment A (150 r.) had a lower chiasma frequency than the other individual sampled at the same time $(12.50$; cf. $13 \cdot 35)$.

Although no really significant increase in chiasma frequency other than that on days 1-3 was recorded, a study of fig. 1 and table 5 does reveal an upward trend about day $6 \frac{1}{2}$. Indeed the mean cell chiasma frequency of the irradiated material at this time is significantly greater than the pooled control mean $(0.01<\mathrm{P}<0.02)$. This increase appears at about the time at which cells irradiated during leptotene-early zygotene would be expected to arrive at diplotene-diakinesis.

The response of the six different bivalent classes scored is shown in fig. 2 . Chiasma scores for the three bivalents $\mathrm{M}_{6}, \mathrm{M}_{7}$ and $\mathrm{S}_{8}$ have been pooled, since all three continued to form a single chiasma under all conditions. It appears, therefore, that the overall changes in mean cell chiasma frequency are the result of changes in the two groups of metacentric chromosomes $\mathrm{L}_{1+2}$ and $\mathrm{L}_{3}$ and in the telocentric group $\mathrm{M}_{4+5}$. All three groups agree in showing 
the increase on days 1 to 3 , though the increase above the control level is slightly more marked in the long chromosomes. In a similar way the decrease seen on days $7 \frac{1}{2}-8 \frac{1}{2}$ is much more pronounced in the metacentric groups $L_{1+2}$ and $L_{3}$, there being little if any decrease in the $M_{4+5}$ bivalents.

\section{Discussion}

Several previous studies have indicated the presence of discrete periods in meiosis of a number of organisms at which recombination or chiasma frequency can be altered by both physical and chemical treatments (see table 5, Westerman, loc. cit., for summary). The pattern of response of chiasma frequency to X-irradiation seen in the present experiment was broadly similar to that seen in $S$. gregaria but does differ in a number of ways. Both $C h$. brunneus and $S$. gregaria agree in showing a significant reduction in chiasma frequency following irradiation of premeiotic DNA synthesis. It is likely that they also agree in showing an increase following irradiation of leptotene-early zygotene, though in Chorthippus the increase is small and seen only on day $+6 \frac{1}{2}\left(t_{\infty}=2.5365 \mathrm{P}=0 \cdot 02-0.01\right)$. Using another grasshopper species, Melanoplus femur-rubrum, Church and Wimber (1969) have also noted an increase in chiasma frequency following irradiation at this time. In their experiment the increase was seen in spite of an overall reduction in chiasma frequency brought about by a $42^{\circ} \mathrm{C}$. temperature regime. The most likely target of treatments given at this time in meiosis is the pairing of homologues. This suggestion is strongly supported by the work of Moens (1970). In an elegant experiment Moens has shown that in Locusta migratoria the pairing of homologues as marked by formation of synaptinemal complexes takes place some 24-48 hours after the completion of premeiotic DNA synthesis.

The patterns of response differ, however, in that Ch. brunneus shows a marked increase in chiasma frequency following irradiation of zygoteneearly pachytene - the classical time of chiasma formation. Similar increases in chiasma frequency following treatments given at this time in meiosis have been noted by Lawrence (loc. cit.) and Craig Cameron (1970).

As with the desert locust, the effect of the $\mathrm{X}$-irradiation appears to be restricted to the longer chromosomes of the complement irrespective of whether these are metacentric or telocentric in organisation. Indeed, the increase or decrease is most pronounced in the longer chromosomes. Thus, following treatment with $150 \mathrm{r}$. X-rays, all of the longer chromosomes are capable of forming more chiasmata, though any decrease is to a minimum of one chiasma per bivalent. Under the experimental conditions used, once this minimum is reached the bivalent continues to form its single chiasma. This finding is in marked contrast to those of Henderson (1966) and Peacock (1968). Following heat shocks of $40^{\circ} \mathrm{C}$. and $37^{\circ} \mathrm{C}$. given to $S$. gregaria and Goniaea australasiae respectively, these two authors showed that the observed reduction in mean cell chiasma frequency was accompanied by an increase in the level of univalence. It is of interest to note that in their heat shock experiment, Ghurch and Wimber (loc. cit.) failed to observe univalents in any of the individuals sampled. As a result of this finding, these authors postulated the existence of two quantitatively different types of chiasmata. Such a conclusion is however unnecessary. Since chiasma formation at 
zygotene-early pachytene is the end-product of an interrelated series of events, each known to be under both major and minor genetic control (Rees, 1961), then the different results may be simply a reflection of different agents affecting these control mechanisms differentially. Thus X-rays and Actinomycin-D might influence chiasma frequency by affecting the "fine" controls of chiasma formation-those governing the distribution of extra chiasmata in the cell over and above the one per bivalent necessary for normal meiotic segregation. Heat shock in the experiments of Henderson and Peacock, on the other hand, seems to affect also those processes which control the formation of this primary chiasma probably through a more direct effect on the pairing of homologues. Just as different genes can affect the coarse and fine controls of chiasma formation, so too can external agents, both physical and chemical.

\section{Summary}

1. The pattern of response of chiasma frequency to X-irradiation was studied in the grasshopper Chorthippus brunneus. In order to relate any observed change to the time of induction, cells undergoing DNA synthesis at the time of irradiation were labelled with $\mathrm{H}^{3}$-Thymidine. Tritiated thymidine alone had no effect on mean cell chiasma frequency.

2. Two distinct radiosensitive periods were found. X-irradiation at the first of these, corresponding with premeiotic DNA synthesis, led to a significant reduction in mean cell chiasma frequency when scored at diplotenediakinesis. The same treatment when given at the time of zygotene-early pachytene gave a significant increase. A slight increase was also noted following X-irradiation given during leptotene-early zygotene.

3. All alterations in mean cell chiasma frequency were the result of changes in the number of chiasmata formed by the longer chromosomesboth metacentrics and telocentrics. No univalents were observed and three smallest bivalents continued to form a single chiasma at all times.

4. It is suggested that different experimental agencies may affect the control mechanisms of chiasma formation differentially. In some organisms a particular treatment may affect only the "fine " controls of chiasma formation, in others the same treatment may also affect the "coarse" controls.

Acknowledgments. - I am grateful to Dr Gareth Jones for many useful discussions, to Dr Mary Jones for help with the analysis of the data and to Miss Patricia Wilkinson for her technical asisstance.

\section{REFERENGes}

CHURCH, K., AND WIMber, D. E. 1969. Meiosis in the grasshopper: Chiasma frequency after elevated temperature and X-rays. Can. F. Genet. Cytol., 11, 209-216.

CRAIG-CAMERON, T. A. 1970. Actinomycin-D and chiasma formation in Schistocerca gregaria Forskål). Chromosoma (Berl.), 30, 169-179.

Henderson, s. A. 1966. Time of chisma formation in relation to the time of DNA synthesis. Nature (Lond.), 211, 1043-1047.

JOHN, B., AND HEWITT, G. M. 1966. Karyotype stability and DNA variability in the Acrididae. Chromosoma (Berl.), 20, 155-172. 
LAWRENCE, C. W. 1961a. The effect of irradiation on different stages of microsporogenesis on chiasma frequency. Heredity, 16, 83-89.

LAWRENCE, C, w. 1961 b. The effect of radiation on chiasma formation in Tradescantia. Radiat. Bot., 1, 92-96.

MOENS, P. B. 1970. Premeiotic DNA synthesis and the time of chromosome pairing in Locusta migratoria. Proc. nat. Acad. Sci. (Wash.), 66, 94-98.

PEACOCK, w. J. 1968. Chiasmata and crossing-over. In Replication and Recombination of Genetic Material, pp. 242-252 (ed. W. J. Peacock and R. D. Brock). Australian Academy of Science, Canberra.

REES, H. 1961. Genetic control of chromosome behaviour. Bot. Rev., 27, 288-318.

southern, D. I. 1967. Chiasma distribution in Truxaline grasshoppers. Chromosome (Berl.), 22, 164-191.

Westerman, M. 1967. The effect of X-irradiation on male meiosis in Schistocerca gregaria (Forskal). I. Chiasma frequency response. Chromosoma (Berl.), 22, 401-416. 